\title{
A Novel Extrusion Microns Embossing Method of Polymer Film
}

\author{
Ying Liu ${ }^{1}$, Wenlong Liu ${ }^{1}$, Yajun Zhang ${ }^{{ }^{*}}$, Daming $\mathrm{Wu}^{1}$, Xinliang Wang ${ }^{2}$ \\ ${ }^{1}$ Institute of Plastics Machinery and Engineering, Beijing University of Chemical Technology, Beijing, China \\ ${ }^{2}$ L.K.Group Ningbo L.K. Technology Co. Ltd., Ningbo, China \\ Email: ${ }^{*}$ zhyj@mail.buct.edu.cn
}

Received December 3, 2011; revised February 10, 2012; accepted February 18, 2012

\begin{abstract}
A continuous hot embossing method is reported in this paper. The continuous means that the extruder and the hot embossing equipment is used in series, the polymer film is embossed after extrusion immediately. The basic principle of this method, the key design point and the corresponding operate process of the embossing equipment is described in this paper. The polymer diffuser with micro-lens matrix on its surface is designed and the optical properties are simulated. Then, the diffuser is fabricated by the embossing equipment. The optical properties of the diffuser are tested and compared with the results of the simulation to verify the reproducibility of the equipment. By this method, the diffuser with large area can be fabricated with high precision of graphic replication, high efficiency and low cost.
\end{abstract}

Keywords: Extrusion Microns Embossing; Hot Embossing Equipment; Diffuser; Reproducibility

\section{Introduction}

The hot embossing method is proposed by Chou of Princeton University in the mid of 1990s. Compared with the traditional lithography technology and the electronic etching technology, the diffraction phenomenon that occurs during optical exposure, and scattering phenomenon that occurs during electron-beam exposure are not exist in this technology. By this technology, the resolution of the graphic on the films can reach a few nanometers. Besides, this technology does not need complex equipment, and the cost is low relatively. The mold is used hundred thousand times in this technology and it is very suitable for mass production [1].

The hot embossing method is suitable for the production with small area, and the process is not continuous. This limitation on the area size is due to the high pressures needed for those processes, often bigger than $1 \mathrm{MPa}$. The processes are quite slow, and the equipments and the molds are usually very complex and expensive [2].

Roll-to-roll nano imprinting lithography has become a promising method in the production of large area micro patterns. This process is very similar to the traditional nano imprinting lithography (NIL). However, in this method the flat mold used in NIL is wrapped around a roller. This method has two significant advantages. Firstly, the contact area of the two rollers is a line; it can achieve very high pressures with a low applied force. Secondly, using this method, a small mold is used to cre-

\footnotetext{
"Corresponding author.
}

ate a significantly larger patterned area in a continuous process which can greatly reduce the cost of mould [3-5].

Although the roll-to-roll nano-imprinting lithography has improved the efficiency and reduced the cost of production, there are still some drawbacks. The polymer film used in this method is bought, it need be heated while the embossing. In order to improve the efficiency of the production, a high heating power system is designed to heat the polymer film. The thermal deformation of the polymer film is very likely to occur while heating. And this influences the thickness uniformity of the polymer film and lead to uneven distribution of pressure during embossing. In order to overcome the shortcoming of the roll-to-roll nano imprinting lithography, a continuous hot embossing system with pre-flatten system is designed in this paper. The extrusion machine and the micro embossing equipment are lined in series. In this system, the polymer film is extruded from the extruder, flattened by the roller and feed into the micro-embossing equipment directly. By this, the polymer film does not need be heated to the required process temperature. This save the heating time and energy, improve the efficiency of the manufacture, and eliminate the thermal deformation.

\section{The Principle of Extrusion Microns Embossing}

\subsection{The Basic Principle of the Method}

The rollers used for embossing have temperature control 
system which ensures the precise control of the embossing temperature. At the same time, by control the gap between the two embossing rollers, the pressure that applied on the polymer film can be controlled.

\subsection{The Process of the Extrusion Microns Embossing Method}

The main process of the extrusion microns embossing method is illustrated in Figure 1. The polymer film is extruded from the extruder through the die. Then it is flattened to ensure the uniformity of thickness by two adjusting rollers. At the last, the polymer film is fed into the pressure roller and the embossing roller. The mold is wrapped on the embossing roller with micro structure. Through the pressure effects between these two rollers, the polymer film is embossed and thus the microstructure on the mold be transferred to the surface of the polymer film. After embossing, the polymer film with the micro structure on its surface need be cooled down.

During this period, the polymer film has to maintain good contact with the mold that is wrapped around the embossing roller to ensure the dimensional stability of the micro structure while cooling. Finally, the polymer film with the micro structure on its surface is de-molded by the draught device. The draught device is composed of two rollers, the top one is wrapped with a layer of soft materials to prevent the damage of the micro structure when pulling.

\section{The Design of the Microns Embossing Equipment}

The temperature and pressure are two most important parameters for the embossing process. The temperature control accuracy and the uniformity of pressure play a decisive role in the quality of the products. The key points

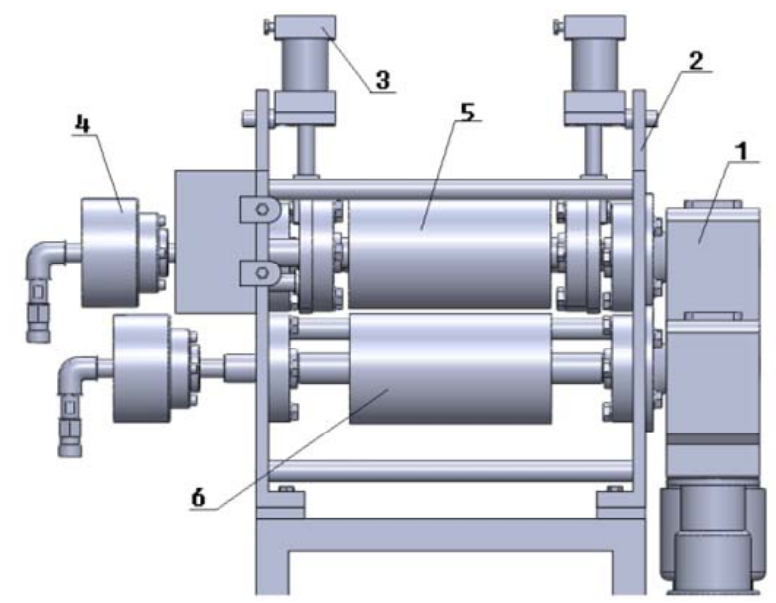

1. Extruder, 2. Die, 3. Film, 4. Pre-compacted thickness control roll, 5. Pressure roller, 6. Imprint roller, 7. Draught device

Figure 1. The schematic of the extrusion microns embossing. of the embossing equipment are focused on the control of the temperature and pressure. Besides, embossing speed, plasticizing quality of the polymer film etc. also have an important impact on the quality of the embossing products.

Figure 2 shows the microns embossing equipment designed in this paper. The main parameters are given in the next chapter in Table 1.

\subsection{Driving System of the Embossing Rollers}

If the speeds of the two rollers are inconsistent, it will lead to a shearing to the polymer film. This will deform the film and thus influence the microstructure transfer accuracy as well as the surface quality of the production. The speed consistency of the pressure roller and embossing roller is very important.

In this paper, the driving system adopts two sets of servo motor driven gear structure to drive the two rollers respectively. The speed of the two rollers servo motor is controlled precisely. The speed consistency of the two rollers is ensured by the closed loop control system of servo motor.

\subsection{Temperature Control System}

The temperature is one of the most important parameters in hot embossing process. If the temperature of the polymer film is too low, it lead to insufficient liquidity of the polymer, incomplete filling of the mold and a large elastic recovery of the micro structure after de-molding; On the contrary, if the temperature of the polymer film is too high, it may destroy the structure of the polymer chain and generate much more defects on the micro structure. Usually, the maximum temperature during embossing process should be controlled at $50^{\circ} \mathrm{C}-100^{\circ} \mathrm{C}$ higher than the $\mathrm{Tg}$ of the polymer. The temperature control system is sensitive to temperature change and has a relatively high heating power so that it response quickly to the temperature change.

Here, the heating oil is used to control the temperature of the two rollers. The oil cycling inside the rollers and its temperature is controlled by a separate machine. By control the temperature of the heating oil, the temperature of the two rollers is controlled. This method has high

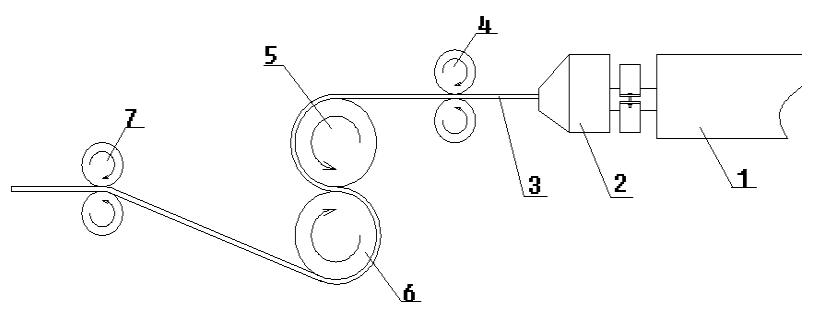

1. Extruder, 2. Die, 3. Film, 4. Pre-compacted thickness control roll, 5. Pressure roller, 6. Imprint roller, 7. Draught device

Figure 2. Hot embossing machine. 
Table 1. The main parameters of the hot embossing machine.

\begin{tabular}{lll}
\hline & Material & Alloy steel \\
Rollers & Diameter $(\mathrm{mm})$ & 140 \\
& Useful Length $(\mathrm{mm})$ & 250 \\
\hline $\begin{array}{l}\text { Adjusting range of the } \\
\text { gap between the two } \\
\text { rollers }(\mathrm{mm})\end{array}$ & Rough adjustment $(\mathrm{mm})$ & $10-30$ \\
\hline $\begin{array}{l}\text { Rotate speed range }(\mathrm{rpm}) \\
\text { Temperature range of the pressure roller }\left({ }^{\circ} \mathrm{C}\right)\end{array}$ & $0-30$ \\
$\begin{array}{l}\text { Temperature range of the embossing roller }\left({ }^{\circ} \mathrm{C}\right) \\
\text { Adjusting range of the pressure }(\mathrm{MPa})\end{array}$ & $20-120$ \\
Embossing speed range $(\mathrm{m} / \mathrm{min})$ & $0-10$ \\
\hline
\end{tabular}

temperature control precision, small temperature fluctuations and a wide range of temperature adjustment.

\subsection{Pressure and Roller Gap Adjustment System}

The pressure is the other important parameter in hot embossing process. If the pressure is too low, it will lead to incomplete filling of the mold. On the other hand, if the pressure is too high, it will generate residual stress in the final products and deformation of the product will occur after de-molding. The uneven of the pressure will lead to poor distribution of the micro structure and make the mold easily damaged. In this paper, the adjustment of the pressure is achieved by adjusting the gap between the two rollers. The roller gap adjustment system includes two parts, the rough and the accurate adjustment mechanism. The rough adjustment mechanism is used to adjust the gap in a wide range, and realize rapid opening and closing of the two rollers. The accurate adjustment mechanism is used for adjust the gap in a very small rang, and control the gap and pressure accurately.

\subsection{The Design of the Embossing Mold}

The micro structure on the roller's surface is transferred to the polymer film directly during the embossing process. Therefore, it decides the shape of the micro structure. In this paper, a chemical etching method is used to produce an array of micro structure on the stainless steel plate. Then the plate is wrapped around the roller. This is illustrated in Figure 3.

The main parameters of this device are given in Table 1.

\section{Simulations and Experimental Analysis}

\subsection{The Simulation of Diffuser's Optical Properties}

The diffuser is mainly used for converting the point light source into surface light source and the light distribute on
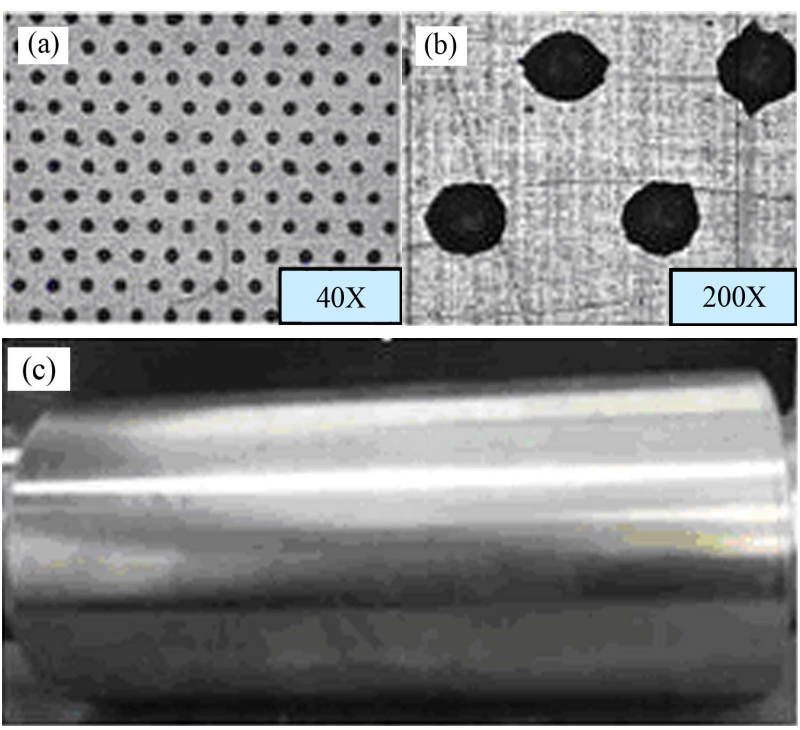

Figure 3. The photo of the roller mold. (a) 40 times photo of the mold; (b) 200 times photo of the mold; (c) Photo of the mold.

its surface uniformly. A key performance of the diffuser is the light distribution uniformity. Generally speaking, the diffuser can be roughly divided into two types, the particle diffusion and surface diffusion. In this paper, the surface diffusion is used and the micro lens matrix is made on the surface of polymer film to achieve the effect of light diffusion. The micro lens matrix is produced by the method that mentioned above. By the light tools software, the optical properties of the diffuser are simulated with the micro lens matrix on its surface. And it is compared with the results without micro structure on its surface. Also, the properties with different micro lens matrix on its surface are also compared. The results are given as follow:

(1) Film without micro structure on its surface. Degree of uniformity is $69.40 \%$ and the Luminousness is $90.44 \%$. The simulation result of the illumination distribution is shown in Figure 4(a).

(2) Polypropylene sheet with micro lens matrix on its surface. Diameter of the micro lens is $175 \mu \mathrm{m}, 400 \mu \mathrm{m}$ pitch, and triangle arrangement. Degree of uniformity is $69.67 \%$, Luminousness is $81.33 \%$. The simulation result of the illumination distribution is shown in Figure 4(b).

(3) The simulation of the Polypropylene sheet with micro lens matrix on its surface. Diameter of the micro lens is $270 \mu \mathrm{m}$, and pitch is $500 \mu \mathrm{m}$, arrangement is triangle. Degree of uniformity is $72.98 \%$, Luminousness is $80.22 \%$. The simulation result of the illumination distribution is shown in Figure 4(c).

From the simulation results and illumination distribution figures, uniformity of light has improved when the polypropylene film has micro lens matrix on its surface. The micro lens matrix on the surface of the polymer film 


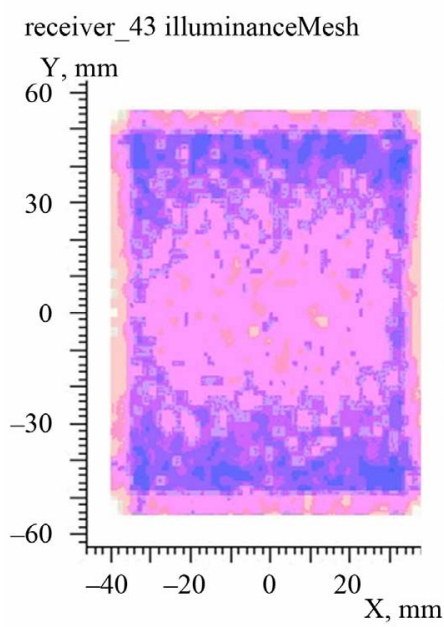

receiver 43 illuminanceMesh

(a)
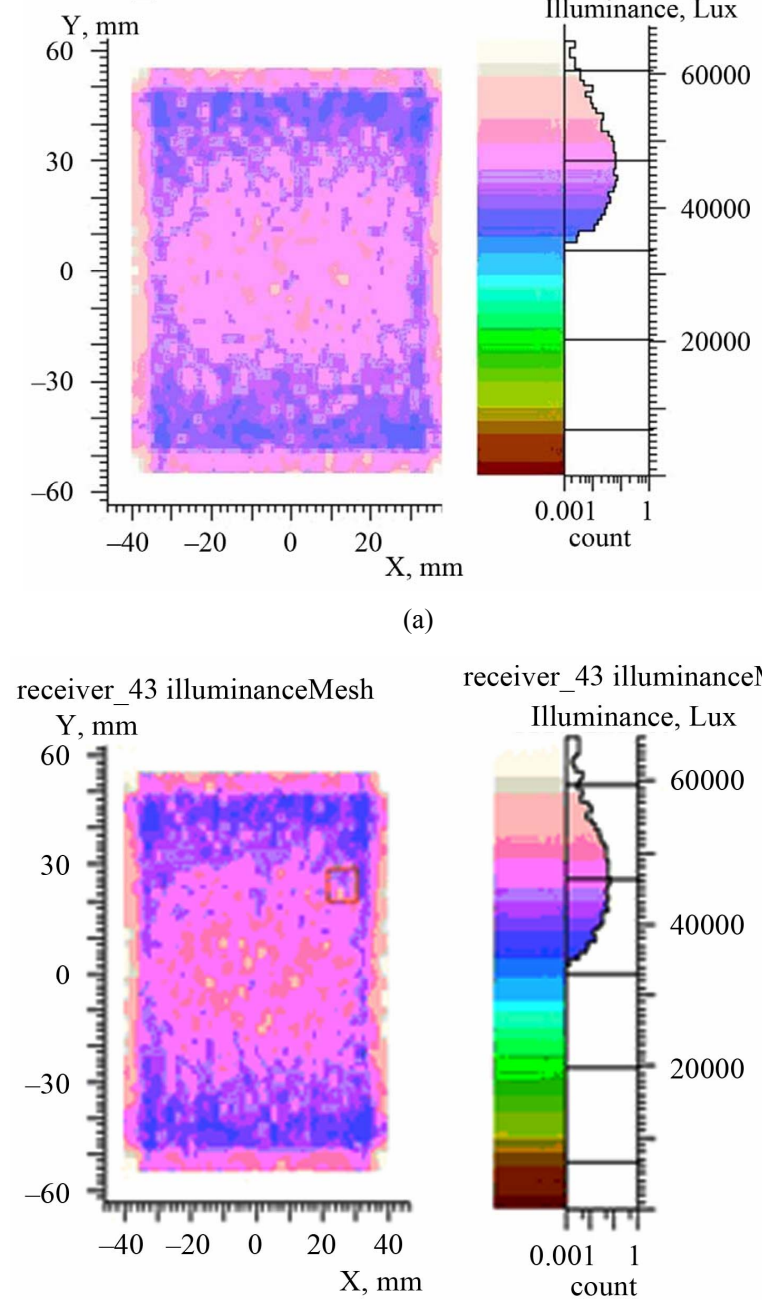

receiver_43 illuminanceMesh Illuminance, Lux

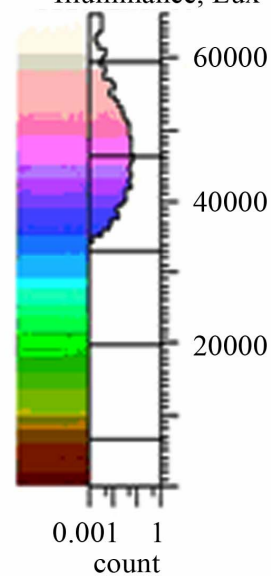

(b)

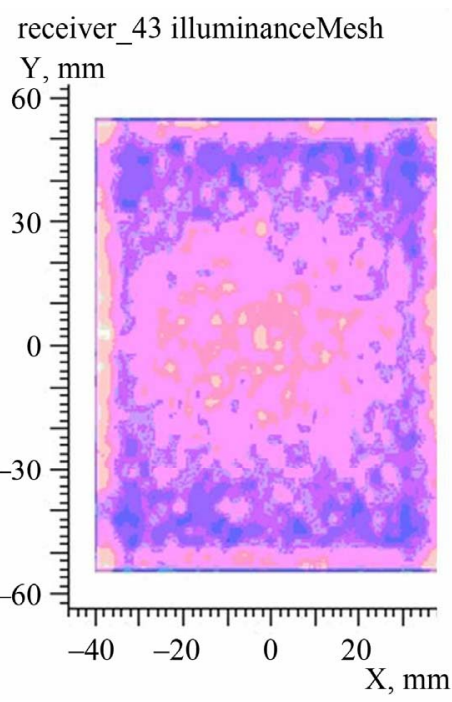

receiver_43 illuminanceMesh

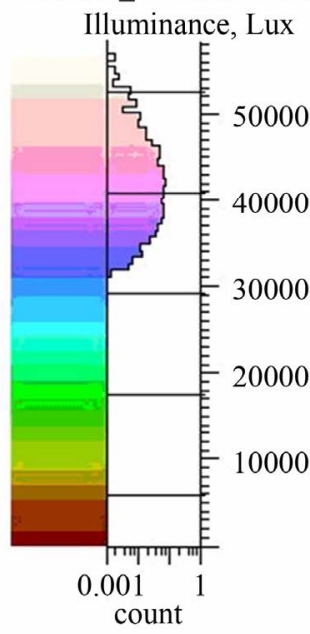

(c)

Figure 4. Illumination distribution of PP film. (a) Without micro structure; (b) With micro structure (diameter of $175 \mu \mathrm{m}$; $400 \mu \mathrm{m}$ pitch); (c) With microstructure (diameter of $270 \mu \mathrm{m} ; 500 \mu \mathrm{m}$ pitch). 
plays an important role in the proliferation of light. Besides, the proliferation of light has a close relationship with the structure of the microstructure that on the surface of the polymer film.

\subsection{Experimental Materials and Equipment}

The Materials used in the experiment is Polypropylene (T4802) of Sinopec Beijing Yanshan Company. The Precision single screw extruder used in the experiment is PSJ-32-28A which is made by Plastic Machinery Research Institute of Beijing University of Chemical Technology. The optical microscope is SZM-B2 which is made by Yongfeng electro mechanical technology company. Light transmittance haze Tester is WGT-S which is made by Shanghai precision scientific instrument Company. Illumination meter is ST-80C which is made by photo electric instrumental Company.

\subsection{Experiments}

First, the parameters of the extruder are adjusted to the required value. At the same time, the hot embossing equipment is started and the embossing parameters are adjusted to ensure the quality of the polymer film. After the extrusion of the film and all the parameters are stable, the polymer film is extruded from the extruder, and fed into the embossing equipment. In this stage, the polymer film is embossing at a low pressure. The polymer film is embossing at a relative large roller gap, so the parameters are adjusted easily. After the embossing process, the parameters are adjusted to the desired value and begin the experiment.

\subsection{Results and Discussion}

\subsubsection{Preparation of Diffuser}

The experimental process condition for the preparation of the diffuser is as follow. The temperature of each section of the extruder is $175^{\circ} \mathrm{C}, 190^{\circ} \mathrm{C}, 200^{\circ} \mathrm{C}, 205^{\circ} \mathrm{C}$ and $210^{\circ} \mathrm{C}$. The temperature of the die is $210^{\circ} \mathrm{C}$. The speed of the embossing roller is $2 \mathrm{rpm}$. The embossing force is 8.2 $\mathrm{KN}$. The temperature of the embossing roller is $60^{\circ} \mathrm{C}$. The temperature of the pressure roller is $165^{\circ} \mathrm{C}$. The diameter of the micro structure on the molds is $150 \mu \mathrm{m}$ and $250 \mu \mathrm{m}, 400$ pitches and 500 pitches respectively. The micro structure is all through-hole and present triangle arrangement on the molds. Because the mold is made by the method of chemical etching, there are some deviations between actual size and design size. The diameter of the actual size of the micro structure is $175 \mu \mathrm{m}$ and $270 \mu \mathrm{m}, 400 \mu \mathrm{m}$ pitches and 500 pitches respectively. The diffuser made by this method is shown in Figure 5. From the picture, we can see that the micro structure on the mold has been successfully transferred to the surface of the polymer film.

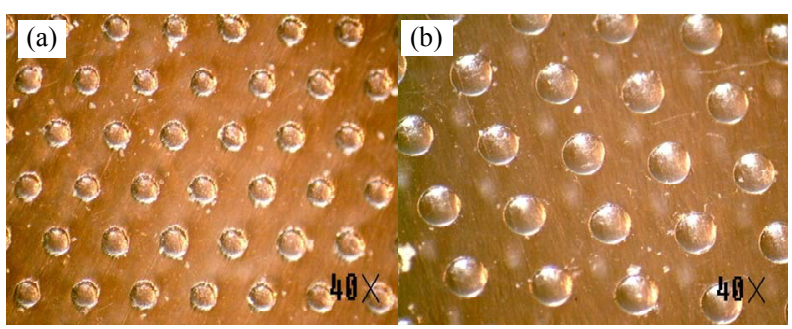

Figure 5. The photo of polymer diffuser.

\subsubsection{The Experimental Test of Diffuser's Optical Properties}

The optical properties of the diffuser are tested by light transmittance/haze Tester and illumination meter. The diffuser has the micro lens matrix on its surface which is made by the method that mentioned above. The test results are shown in Table 2.

An optical test platform is used to test the diffuser's effect on the proliferation of light. This optical test platform include a laser pointer light, a support frame used for fixing laser pointer light and diffuser, a white screen used to receive and display the effect of proliferation of light. The experimental results are shown in Figure 6. From the experimental results, the proliferation and uniformity of light is improved by the micro lens matrix on its surface.

\section{Conclusion}

A continuous polymer micro embossing method and its principle are introduced in this paper. The critical parameters during the embossing process and the influence of these parameters on the accuracy of graph transfer are analyzed. Then, hot embossing equipment is designed to meet the requirement of the process conditions. Using this hot embossing machine and a precision single screw extruder, the polymer diffuser with micro lens matrix on

Table 2. The optical properties of the diffuser.

\begin{tabular}{cccc}
\hline & Luminousness & Uniformity & Haze \\
\hline PP film & 88.1 & 65.9 & 26.0 \\
PP 0.175 & 82.5 & 69.6 & 34.6 \\
PP 0.27 & 85.7 & 73.6 & 37.3 \\
\hline
\end{tabular}
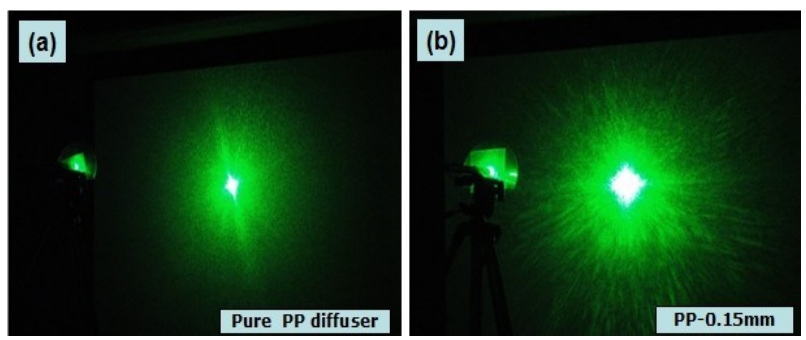

Figure 6. The diffusion effect chart of the diffuser. 
its surface is produced successfully. The optical properties of the polymer diffuser are analyzed though simulations and experiments. The results show that the micro structure on the mold was perfectly transferred to the polymer film and the diffuser's light uniformity simulation results are consistent with the experimental results. This is a feasible method for producing polymer diffuser with micro structure on the surface. This is a very promising method in the field of making micro structure. It has the advantage of high precision, simple process, easy to operate, high production efficiency and low cost.

\section{REFERENCES}

[1] M. D. Fagan, B. H. Kim and D. G. Yao, "A Novel Process for Continuous Thermal Embossing of Large-Area Nanopatterns onto Polymer Films," Advances in Polymer Technology, Vol. 28, No. 4, 2009, pp. 246-256. doi:10.1002/adv.20167
[2] S.-W. Youn, M. Ogiwara, H. Goto, M. Takahashi and R. Maeda, "Prototype Development of a Roller Imprint System and Its Application to Large Area Polymer Replication for a Microstructured Optical Device," Journal of Materials Processing Technology, Vol. 202, No. 1-3, 2008, pp. 76-85. doi:10.1016/j.jmatprotec.2007.08.069

[3] S. H. Ahn and L. J. Guo, "Large-Area Roll-to-Roll and Roll-to-Plate Nanoimprint Lithograp: A Step toward HighThroughput Application of Continuous Nanoimprinting," ACS Nano, Vol. 3, No. 8, 2009, pp. 2304-2310. doi: $10.1021 / \mathrm{nn} 9003633$

[4] S. H. Ahn and L. J. Guo, "High-Speed Roll-to-Roll Nanoimprint Lithography on Flexible Plastic Substrates," Advanced Materials, Vol. 20, No. 11, 2008, pp. 2044-2049. doi:10.1002/adma.200702650

[5] S. H. Lan, J.-H. Song, M. G. Lee, J. Ni, N. K. Lee and H.-J. Lee, "Continuous Roll-to-Flat Thermal Imprinting Process for Large-Area Micro-Pattern Replication on Polymer Substrate," Microelectronic Engineering, Vol. 87 , No. 12, 2010, pp. 2596-2600. 